\title{
Tablet Splitting Performance Evaluation of Losartan Potassium and Olmesartan Medoxomil IR Tablets Marketed in Bangladesh
}

\author{
Saimon Shahriar ${ }^{1}$, Abu Asad Chowdhury ${ }^{2}$, Md. Shah Amran ${ }^{2}$ \\ and Jakir Ahmed Chowdhury ${ }^{3}$
}

\author{
${ }^{1}$ Department of Pharmacy, Faculty of Pharmacy, University of Dhaka Dhaka-1000 Bangladesh \\ ${ }^{2}$ Department of Pharmaceutical Chemistry, Faculty of Pharmacy, University of Dhaka \\ Dhaka-1000, Bangladesh \\ ${ }^{3}$ Department of Pharmaceutical Technology, Faculty of Pharmacy, University of Dhaka \\ Dhaka-1000, Bangladesh
}

(Received: Jun 1, 2020; Accepted: July 16, 2020; Published (web): July 25, 2020)

\begin{abstract}
Tablet splitting is a widely used practice in Bangladesh but there are no regulations for pharmaceutical companies to ensure that tablets are manufactured in such a way that facilitates appropriate splitting. Seven Losartan Potassium (LSP) brands and five Olmesartan Medoxomil (OLM) brands were selected and necessary number of tablets as well as a tablet splitter was collected. Weight variation, assay, invitro dissolution and loss of weight parameters were evaluated according to recent guidelines on the tablet fractions split by hand and by the splitter separately. Seven out of twelve brands displayed acceptable results in all parameter evaluations. EP and USP standards produced dissimilar results. In all assessments, machine-split portions produced better results than hand-split ones and so, a tablet splitter was found to be more reliable than using hands only. This study is preliminary in nature and thus further extensive studies are in progress involving more samples, more personnel and more laboratories as well as utilizing properly validated methodology and precisely calibrated instruments to get more conclusive data.
\end{abstract}

Key words: Tablet splitting, tablet splitter, Losartan Potassium, Olmesartan Medoxomil, guidelines.

\section{Introduction}

Tablet is a widely used and popular dosage form intended for oral ingestion and there are many tablets available with single or multiple scoring lines on upper or lower surface in order to ease the splitting of a tablet into two or more equal fractions (Ono et al., 2013). Tablet splitting is an admissible practice (Teixeira et al., 2017) for the treatment of children suffering from congenital diseases like congenital Addison's disease or atrial septal defect (Madathilethu et al., 2018). Tablet splitting is also practiced in order to reduce the cost of treatment (Quinzler et al., 2006) or when the smaller dose is not available nearby, which is a common situation in rural area in Bangladesh where availability of medicines is low and the majority of people have no affordability (Kasonde et al., 2019). Tablet splitting is common in case of immediate release drugs but not recommended in case of modified release drugs like birth control pills (Trivedi et al., 2017). Advantages of scored tablets include ease of swallowing and cost reduction (Carr-Lopez et al., 1995). Scored tablets also come in handy when the patient is on an increasing or a decreasing dosing schedule and needs different doses at different times (Van Santen et al., 2002). This practice has some drawbacks too, including generation of unequal portions (Zaid and Ghosh, 2011), loss of mass due to fragmentation and powdering (Van Santen et al., 2002) and affecting brand reputation (Stimpel et al., 1985). In order to

Corresponding author: Jakir Ahmed Chowdhury; Tel.: +8801711043535; Email: jakir@ du.ac.bd

DOI: https://doi.org/10.3329/bpj.v23i1.48335 
overcome these problems, various means have been suggested including appropriate patient counseling (Wilson et al., 1996), using a tablet splitter as well as formulating tablets in such a way that facilitates tablet splitting (e.g. manufacturing tablets in oblong shape) (Myriam et al., 1994).

Losartan Potassium (LSP) and Olmesartan Medoxomil (OLM), Angiotensin-II Receptor Blocker (ARB), competitively bind with Angiotensin-II type $1\left(\mathrm{AT}_{1}\right)$ receptor resulting in selective and powerful inhibition of Angiotensin-II which is a causative factor of hypertension and thus relieve the symptoms of hypertension (Aniñon et al., 2014). The usual doses of LSP are 25-, 50- and 100-mg and that of OLM are 10-, 20- and 40-mg. For children with low body weight and for patients with renal impairment or intravascular volume depletion, low doses of LSP or OLM may be required and hence, scored tablets may be needed (Muir and Keating, 2010; Shahinfar et al., 2005).

The aim of this study was to evaluate the functionality of scoring lines of commonly available LSP and OLM tablet brands where the tablets were scored by hands as well as by using a tablet cutter. Various parameters like weight variation, assay, dissolution etc. were analyzed for different doses and brands of LSP and OLM following EP, USP and FDA guidelines. The necessity of implementing those guidelines in the local market of Bangladesh has also been discussed on the basis of the results of this study.

\section{Materials and Methods}

Selection of tablets for testing and collection of LSP and OLM reference standards: There are 59 LSP- and 14 OLM-brands available in Bangladesh. Among them 5 LSP- and 3 OLM-brands were randomly selected and collected from medicine shops all over Dhaka. The tablet brands were randomly coded: LSP-1, LSP-2, LSP-3, LSP-4 and LSP-5 for Losartan Potassium brands and OLM-1, OLM-2 and OLM-3 for Olmesartan Medoxomil brands. Both (50and 100-mg) doses LSP-1 and LSP-2 were analyzed while the rest were of $50 \mathrm{mg}$ dose. On the other hand,
20 and $40 \mathrm{mg}$ doses of both OLM-1 and OLM-2 were studied while OLM-3 was of only $20 \mathrm{mg}$ dose. A tablet splitter, Sciencemate, was used for instrumental scoring of tablets.

Preparation of standard solution for assay and in vitro dissolution study: Standard solutions of LSP and OLM reference standards were prepared following recognized and validated protocols (Aniñon et al., 2014; Celebier and Altinoz, 2007). Appropriately diluted solutions were prepared and used to construct standard curves at $205 \mathrm{~nm}\left(\mathrm{R}^{2}=\right.$ 0.997) and at $258 \mathrm{~nm}\left(\mathrm{R}^{2}=0.996\right)$ for LSP and OLM, respectively.

Weight variation: Weight variation of the tablets was measured following EP and USP standards separately and then the results have been displayed in a table. Percent loss of weight was also calculated LSP and OLM whole tablets and split portions were assayed following the well-established methods (Aniñon et al., 2014; Celebier and Altinoz, 2007).

Assay: 20 whole tablets and 20 tablet portions were assayed for each brand. For every analysis, tablets were broken and powdered with a mortar and pestle and an aliquot powder that is supposed to contain specific amounts of the API was measured and assayed.

Dissolution: Dissolution testing was performed following the USP standards. For LSP tablets, $900 \mathrm{ml}$ water was the medium, apparatus was type 2 (paddle) with $50 \mathrm{rpm}$ and Q value was $75 \%$ in 30 minutes. For OLM tablets, everything was same except the medium was $900 \mathrm{ml}$ phosphate buffer with $\mathrm{pH} 6.8$. All analyses were done on hand-split and machinesplit tablets individually.

Loss of weight: 30 whole tablets and the portions from those tablets were measured for loss of weight following established procedures. Hand-split and machine-split portions were checked separately.

\section{Results and Discussion}

Weight variation: The test results are displayed in table 1 . Weight variability of resulting fragments has been reported as significant and extensive in previous studies (Stimpel et al., 1984; Gupta and 
Gupta, 1988). In our study, we found similar results in some cases and better results in case of the rest.

Seven brands of Losartan potassium (LSP-1 50and 100-mg, LSP-2 50- and 100-mg, OLM-1 20- and 40-mg and OLM-2 $40 \mathrm{mg}$ ) showed results inside the acceptance criteria according to both the EP and USP. On the other hand, LSP-4 (50 mg), LSP-5 (50 $\mathrm{mg}$ ) and OLM-3 (20 mg) showed unacceptable results as per both the EP and USP standards. Though only one tablet fraction outside $15 \%$ of the mean weight are acceptable by EP but 7 fractions of LSP-4 $(50 \mathrm{mg}), 11$ fractions of LSP-5 $(50 \mathrm{mg})$ and 8 fractions of OLM-3 (20 mg) were outside >15\% whereas 3 fractions, 4 fractions and 4 fractions were outside $25 \%$ of the expected weight in case of LSP-4 $(50 \mathrm{mg})$, LSP-5 $(50 \mathrm{mg})$ and OLM-3 $(20 \mathrm{mg})$ tablets, respectively, whereas no fraction was allowed according to USP.

Table 1. Weight variation test result (hand-split) and comparison between EP and USP criteria.

\begin{tabular}{|c|c|c|c|}
\hline \multirow[t]{2}{*}{ Tablet brand } & \multicolumn{2}{|c|}{$\begin{array}{l}\text { No. of half-tablets deviating by } \\
\text { (EP criteria) }\end{array}$} & \multirow{2}{*}{$\begin{array}{c}\text { No. of half-tablets deviating by } \\
\text { (USP criteria) }\end{array}$} \\
\hline & $>15 \%$ of $\mathrm{MW}$ & $>25 \%$ of $\mathrm{MW}$ & \\
\hline LSP-1 $50 \mathrm{mg}$ & 0 & 0 & 0 \\
\hline LSP-1 100 mg & 0 & 0 & 0 \\
\hline LSP-2 $50 \mathrm{mg}$ & 1 & 0 & 0 \\
\hline LSP-2 $100 \mathrm{mg}$ & 0 & 0 & 0 \\
\hline LSP-3 $50 \mathrm{mg}$ & 2 & 1 & 1 \\
\hline LSP-4 $50 \mathrm{mg}$ & 7 & 3 & 3 \\
\hline LSP-5 $50 \mathrm{mg}$ & 11 & 4 & 4 \\
\hline OLM-1 20 mg & 0 & 0 & 0 \\
\hline OLM-1 40 mg & 0 & 0 & 0 \\
\hline OLM-2 $20 \mathrm{mg}$ & 2 & 1 & 1 \\
\hline OLM-2 $40 \mathrm{mg}$ & 1 & 0 & 0 \\
\hline OLM-3 $20 \mathrm{mg}$ & 8 & 4 & 4 \\
\hline Total acceptable & \multicolumn{2}{|c|}{$7 / 12$} & 9/12 \\
\hline
\end{tabular}

$\mathrm{MW}=$ mean weight EW = expected weight.

As all the tablets were split by the same person in identical situation, the human factor might not be the cause of the unacceptable result of these three brands unlike in the case reported by Zaid and Ghosh (2011). Therefore, the only possible cause for this result could be the formulation which might not be up to the mark to ensure appropriate splitting. Formulation can be improved for the purpose of better splitting by producing a deeper scoring line and making the tablets in oblong or elongated shapes (Gupta and Gupta, 1988). LSP-3 (50 mg) and OLM-2 $(20 \mathrm{mg})$ showed results which were acceptable by USP standards but not acceptable by EP standards. This showed that same tablets could be deemed fit or unfit by different regulatory standards resulting in manufacturing companies being forced to follow varying regulatory standards.

Although both the EP and USP specified the use of hands while testing scored tablets, however, a tablet cutter was used to determine whether it improved the results. The results are displayed in table 2. LSP-5 (50 mg) and OLM-3 (20 mg) still showed unacceptable results by both EP and USP standards. However, the number of fractions outside the acceptable limit reduced. LSP-5 $(50 \mathrm{mg})$ revealed 9 fractions outside $15 \%$ of the mean weight in case of machine-split tablets, whereas in hand-splitting this number was 11 fractions. Similarly, OLM-3 (20 mg) had 7 fractions outside $15 \%$ of the mean weight in case of machine-split tablets, whereas in hand- 
splitting this number was 8 fractions. Similar decrease was seen in fraction numbers outside $25 \%$ of the mean weight too. Although LSP-4 $(50 \mathrm{mg})$ tablets produced unacceptable results in case of handsplit tablets by both EP and USP standards, this brand showed acceptable results using machine-splitting tablets by USP standards. The reason for this improvement could only be the use of a tablet splitter, as all the other factors were kept unchanged.
Lastly, the total number of acceptable brands was 7 (according to EP) and 9 (according to USP) in case of hand-split tablets. But in case of machine-split tablets, this number was increased to 8 (according to EP) and 10 (according to USP). From these data, it was unambiguous that machine-splitting improved splitting performance, which was in line with previous findings (Sedrati et al., 1994).

Table 2. Weight variation test result (machine-split) and comparison between EP and USP criteria.

\begin{tabular}{|c|c|c|c|}
\hline \multirow[t]{2}{*}{ Tablet brand } & \multicolumn{2}{|c|}{$\begin{array}{l}\text { No. of half-tablets deviating by } \\
\text { (EP criteria) }\end{array}$} & \multirow{2}{*}{$\begin{array}{c}\begin{array}{c}\text { No. of half-tablets deviating by } \\
\text { (USP criteria) }\end{array} \\
>25 \% \text { of EW }\end{array}$} \\
\hline & $>15 \%$ of $\mathrm{MW}$ & $>25 \%$ of $\mathrm{MW}$ & \\
\hline LSP-1 $50 \mathrm{mg}$ & 0 & 0 & 0 \\
\hline LSP-1 100 mg & 0 & 0 & 0 \\
\hline LSP-2 $50 \mathrm{mg}$ & 0 & 0 & 0 \\
\hline LSP-2 100 mg & 0 & 0 & 0 \\
\hline LSP-3 $50 \mathrm{mg}$ & 1 & 0 & 0 \\
\hline LSP-4 $50 \mathrm{mg}$ & 6 & 2 & 2 \\
\hline LSP-5 $50 \mathrm{mg}$ & 9 & 4 & 4 \\
\hline OLM-1 20 mg & 0 & 0 & 0 \\
\hline OLM-1 $40 \mathrm{mg}$ & 0 & 0 & 0 \\
\hline OLM-2 $20 \mathrm{mg}$ & 1 & 1 & 1 \\
\hline OLM-2 $40 \mathrm{mg}$ & 1 & 0 & 0 \\
\hline OLM-3 $20 \mathrm{mg}$ & 7 & 3 & 3 \\
\hline Total acceptable & \multicolumn{2}{|c|}{$8 / 12$} & $10 / 12$ \\
\hline
\end{tabular}

$\mathrm{MW}=$ mean weight; $\mathrm{EW}=$ expected weight.

Assay: The United States FDA states that the split portions of tablets need to pass the same finished product tests mentioned for whole tablets in USP. Assay was done to see whether split portions in this study passed those criteria. The results are presented in table 3.

Acceptance criteria for LSP and OLM were 95$105 \%$ and $93-105 \%$ of the claimed API amount respectively, according to USP. LSP-1 (50- and 100$\mathrm{mg}$ ), LSP-2 (50- and 100-mg), OLM-1 (20- and 40$\mathrm{mg})$ and OLM-2 $(40 \mathrm{mg})$ tablets showed results that were inside the USP acceptance criteria. The assay values ranged from $95.48 \%$ to $97.94 \%$ in case of LSP brands and $93.15 \%$ to $97.5 \%$ in case of OLM. LSP-3 (50 mg), LSP-4 (50 mg), LSP-5 (50 mg), OLM-2 (20 $\mathrm{mg})$ and OLM-3 (20 mg) showed assay results as $92.44 \%, 90.04 \%, 84.72 \%, 91.1 \%$ and $86.4 \%$, respectively, which made these brands outside the acceptance criteria specified by USP. Human factor might not be responsible for these abysmal results as some tablets produced the acceptable results, which were also split by the same person in similar situation. Therefore, formulation might be responsible for those unacceptable results.

After comparing the results of weight variation study and assay, it was seen that LSP-3 $(50 \mathrm{mg})$ and OLM-2 $(20 \mathrm{mg})$ tablets showed acceptable results in weight variation study but failed to show acceptable values during assay. It indicated that these two brands were not actually split appropriately but would have 
been deemed fit by USP standards if weight variation test was done only, not the assay. However, assay has not been made mandatory by USP or EP. As per the FDA guidelines, all parameters that are necessary to test whole tablets are equally important for split tablet testing. Hence, FDA guidelines may be considered as the most robust guidelines in this context.

Table 3. Assay result of hand-split and machine-split fractions.

\begin{tabular}{|c|c|c|c|c|c|}
\hline \multirow[t]{3}{*}{ Brand name } & \multirow{3}{*}{$\begin{array}{l}\text { Claimed API in } \\
\text { half-tablets (mg) }\end{array}$} & \multicolumn{4}{|c|}{ Assayed drug content in half-tablets } \\
\hline & & \multicolumn{2}{|c|}{ Hand-split } & \multicolumn{2}{|c|}{ Machine-split } \\
\hline & & $\mathrm{Mg}$ & $\%$ & $\mathrm{mg}$ & $\%$ \\
\hline LSP-1 $50 \mathrm{mg}$ & 25 & 24.38 & 97.52 & 24.61 & 98.44 \\
\hline LSP-1 $100 \mathrm{mg}$ & 50 & 48.97 & 97.94 & 49.32 & 98.64 \\
\hline LSP-2 $50 \mathrm{mg}$ & 25 & 23.87 & 95.48 & 24.14 & 96.56 \\
\hline LSP-2 $100 \mathrm{mg}$ & 50 & 48.22 & 96.44 & 48.85 & 97.70 \\
\hline LSP-3 $50 \mathrm{mg}$ & 25 & 23.11 & 92.44 & 23.92 & 95.68 \\
\hline LSP-4 $50 \mathrm{mg}$ & 25 & 22.51 & 90.04 & 22.93 & 91.72 \\
\hline LSP-5 $50 \mathrm{mg}$ & 25 & 21.18 & 84.72 & 22.31 & 89.24 \\
\hline OLM-1 $20 \mathrm{mg}$ & 10 & 9.75 & 97.50 & 9.84 & 98.40 \\
\hline OLM-1 $40 \mathrm{mg}$ & 20 & 19.23 & 96.15 & 19.57 & 97.85 \\
\hline OLM-2 $20 \mathrm{mg}$ & 10 & 9.11 & 91.10 & 9.62 & 96.20 \\
\hline OLM-2 $40 \mathrm{mg}$ & 20 & 18.63 & 93.15 & 18.97 & 94.85 \\
\hline OLM-3 $20 \mathrm{mg}$ & 10 & 8.64 & 86.40 & 8.93 & 89.30 \\
\hline Total acceptable & & \multicolumn{2}{|c|}{$7 / 12$} & \multicolumn{2}{|c|}{ 9/12 } \\
\hline
\end{tabular}

USP specifications included the use of hands while splitting the tablets, but we wanted to investigate the performance of machine-split tablet fractions. The results are also displayed in table 3 . From the table, it was indisputable that the assay values of the machine-split tablets were consistently higher than that of the hand-split tablets. Even, in case of LSP-3 $(50 \mathrm{mg})$ and OLM-2 $(20 \mathrm{mg})$ tablets, the hand-split fractions were found to be outside the USP acceptance criteria $(92.44 \%$ and $91.1 \%$, respectively) but the machine-split fractions showed values inside the criteria (95.68\% and $96.2 \%$, respectively). Finally, the total number of acceptable brands was 7 in case of hand-split tablets but in case of machine-split tablets, this number was increased to 9. From these figures, it was explicit that machinesplitting improved splitting performance, which was similar to the previous findings (Sedrati et al., 1994).

In vitro dissolution study: In vitro dissolution study of the tablet fractions was done following the protocols of USP. The result is displayed in table 4. The tablets are considered to be fit if $75 \%$ of the expected API is dissolved within 30 minutes.

LSP-1 (50- and 100-mg), LSP-2 (50- and 100$\mathrm{mg}$ ), OLM-1 (20- and 40-mg) and OLM-2 (40 mg) tablets showed results that are inside the USP specified acceptance criteria. Their dissolution values varied from $84.64 \%$ to $94.6 \%$ in case of LSP brands and from $78.25 \%$ to $91.6 \%$ in case of OLM brands. LSP-3 (50 mg), LSP-4 (50 mg), LSP-5 (50 mg), OLM-2 (20 mg) and OLM-3 (20 mg) displayed results that are outside the USP acceptance criteria as they showed $70.16 \%, 65.68 \%, 68.84 \%, 68.6 \%$ and $67.4 \%$ dissolution values, respectively within the specific time period. The same person did all the tests in similar conditions and so, personal variation might not be the cause of these unacceptable values. The more probable cause might be that formulations of these tablets were not up to the mark to ensure proper splitting. 
We wanted to explore the performance of machine-split tablet fractions too. The results are also displayed in table 4. From the table, it is evident that the dissolution values of the machine-split tablets were higher than that of hand-split tablets in every case. Even, in case of OLM-2 (20 mg) tablets, the hand-split fractions were outside $(68.6 \%)$ of USP acceptance criteria but the machine-split fractions showed values inside (79.5\%) the criteria. Thus, the total number of acceptable brands was 7 in case of hand-split tablets but it increased to 8 in case of machine-split tablets. From these facts, it is clear that the machine improved splitting performance which was in parallel with the previous studies (Sedrati et al., 1994) and other findings of this study.

Table 4. Dissolution test result according to USP and comparison between hand-split and machine-split tablet fractions.

\begin{tabular}{|c|c|c|c|c|c|c|}
\hline \multirow{3}{*}{ Tablet Brand } & \multicolumn{3}{|c|}{ Hand-split half-tablets } & \multicolumn{3}{|c|}{ Machine-split half-tablets } \\
\hline & \multicolumn{2}{|c|}{$\begin{array}{l}\text { API dissolved in } 30 \\
\text { minutes }\end{array}$} & \multirow[t]{2}{*}{$\begin{array}{l}\text { No. of half-tablets } \\
\text { with <Q-15\% values }\end{array}$} & \multicolumn{2}{|c|}{$\begin{array}{l}\text { API dissolved in } \\
30 \text { minutes }\end{array}$} & \multirow{2}{*}{$\begin{array}{l}\text { No. of half- } \\
\text { tablets with <Q- } \\
15 \% \text { values }\end{array}$} \\
\hline & $\mathrm{mg}$ & $\%$ & & $\mathrm{mg}$ & $\%$ & \\
\hline LSP-1 $50 \mathrm{mg}$ & 23.65 & 94.60 & 0 & 24.12 & 96.48 & 0 \\
\hline LSP-1 $100 \mathrm{mg}$ & 45.62 & 91.24 & 0 & 46.77 & 93.54 & 0 \\
\hline LSP-2 $50 \mathrm{mg}$ & 21.92 & 87.68 & 0 & 22.85 & 91.40 & 0 \\
\hline LSP-2 $100 \mathrm{mg}$ & 42.32 & 84.64 & 0 & 43.11 & 86.22 & 0 \\
\hline LSP-3 $50 \mathrm{mg}$ & 17.54 & 70.16 & 0 & 18.04 & 72.16 & 0 \\
\hline LSP-4 $50 \mathrm{mg}$ & 16.42 & 65.68 & 1 & 17.66 & 70.64 & 0 \\
\hline LSP-5 $50 \mathrm{mg}$ & 17.21 & 68.84 & 3 & 18.88 & 75.52 & 1 \\
\hline OLM-1 $20 \mathrm{mg}$ & 8.64 & 86.40 & 0 & 8.95 & 89.50 & 0 \\
\hline OLM-1 $40 \mathrm{mg}$ & 18.32 & 91.60 & 0 & 19.11 & 95.55 & 0 \\
\hline OLM-2 $20 \mathrm{mg}$ & 6.86 & 68.60 & 0 & 7.95 & 79.50 & 0 \\
\hline OLM-2 $40 \mathrm{mg}$ & 15.65 & 78.25 & 0 & 16.78 & 83.90 & 0 \\
\hline OLM-3 $20 \mathrm{mg}$ & 6.74 & 67.40 & 2 & 7.31 & 73.10 & 1 \\
\hline Total acceptable & \multicolumn{3}{|c|}{$7 / 12$} & \multicolumn{3}{|c|}{$8 / 12$} \\
\hline
\end{tabular}

According to USP, $\mathrm{Q}=75$ in case of both LSP and OLM; Expected drug amount in fragments is half of the label claim of the whole tablets.

Loss of weight: The United States FDA also stated that loss of weight during splitting should not be more than $3 \%$. The results of percent loss of weight of different brands are displayed in figure 1. LSP-1 (50- and 100-mg), LSP-2 (50- and 100-mg), OLM-1 (20- and 40-mg) and OLM-2 (40 mg) tablets demonstrated $2.9 \%, 2.8 \%, 2.1 \%, 2.8 \% 1.9 \%, 2.1 \%$ and $2.5 \%$ loss of weight, respectively. None of these losses was more than 3\%. Therefore, these brands were inside acceptable limit. LSP-3 (50 mg), LSP-4 (50 mg), LSP-5 (50 mg), OLM-2 (20 mg) and OLM$3(20 \mathrm{mg})$ showed $3.6 \%, 4.1 \%, 7.2 \%, 3.3 \%$ and $6.5 \%$ loss of weight, respectively. These tablets were outside acceptable limit. Because of splitting the tablets by the same person in similar conditions, personal variation might not be the reason behind the unacceptable values. Formulation inefficiency might be one of the reasons behind that.

The benefit of using a tablet splitter could easily be noticed in this figure. All the brands showed less loss of weight when the machine was used. Even LSP-3 (50 mg), LSP-4 (50 mg) and OLM-2 (20 mg) tablets showed acceptable loss of weight figures when machine-split portions were used. This finding clearly demonstrated that tablet splitter could be better alternative to hand-splitting. 
Considering the results of all the parameters evaluated in this study, it can be perceived that LSP-3 (50 $\mathrm{mg})$ and OLM-2 (20 mg) tablets showed acceptable results as per the USP weight variation test but failed to show acceptable results in assay and loss of weight. It was stated in FDA guideline that assay and other parameters should be included in
USP split tablet testing regulations (Ciavarella et al., 2016). From our experimental data, it could be stated that loss of weight might also be a valuable testing parameter in this context. This parameter is included in the FDA guidelines, but not included in the EP or USP regulations.

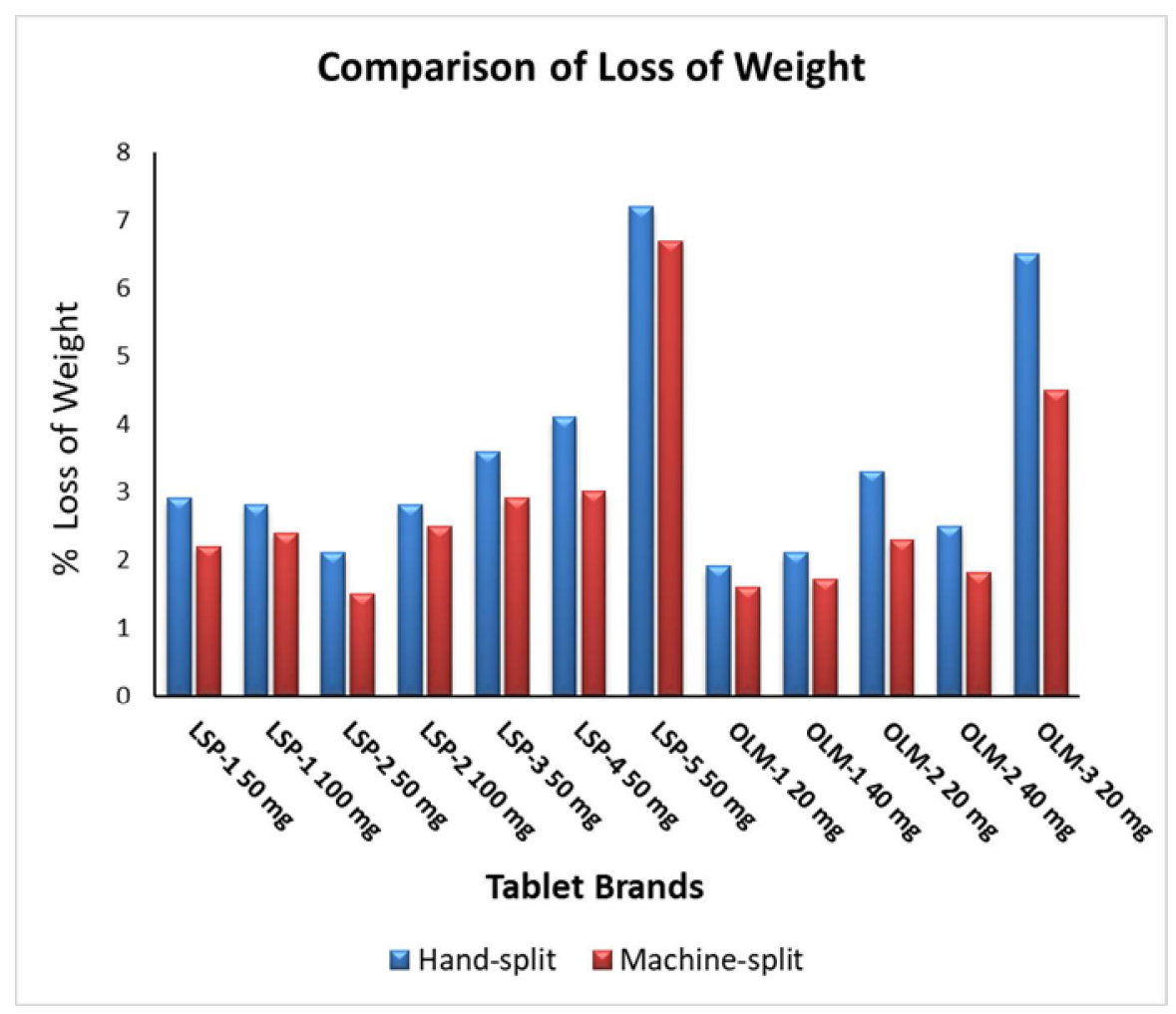

Figure 1. Comparison of loss of weight of tablet brands while splitting.

Out of 12 brands tested, 7 brands showed acceptable results in all the parameters. From the other 5 brands, two brands (LSP-3 $50 \mathrm{mg}$ and OLM-2 $20 \mathrm{mg}$ ) qualified in most of the evaluations when machine-split tablet portions were used but did not qualify when hand-split fractions were used. The other 3 brands displayed abysmal splitting performances in all cases. The formulation of those tablets was probably not favorable for splitting as human error was minimized. Regulations may be modified to adhere to established guidelines regarding tablet scoring. A tablet splitter was found to be more reliable in this study. Several studies reported that splitters were a better alternative
(Sedrati et al., 1994), whereas some studies reported inconclusive results (Gupta and Gupta, 1988). However, majority of studies found that hand splitting causes variation of doses in split fractions (Nimmi et al., 2010). Further extensive studies involving more samples and personnel are essential to get more acceptable data.

\section{Conclusion}

Tablet splitting is often essential all over the world. Lack of medicine shops, unavailability of tablets with appropriate doses and a number of other reasons can compel a patient to split tablets and take 
a fraction. Hence, the knowledge of appropriate splitting is essential for patients, caregivers and associated people. They should be aware of the problems associated with tablet splitting and how to overcome these problems. At the same time, pharmaceutical companies should look into the fact that their products may need to be split before ingestion and the companies should prepare for that. Formulations should be adjusted to ease tablet splitting. Also, providing adequate scoring lines on tablets is the responsibility of the companies. Regulations for scored tablets published by USP, FDA and EP are now available. The DGDA may consider of implementing these regulations in our country. In this study, $58 \%$ of the evaluated brands displayed satisfactory results in all the tests. Tablet cutter was found to be more reliable than handsplitting. Only weight variation and dissolution test were found vary during evaluation of tablet scoring. Tablet cutter is not readily available in Bangladesh and the price is high. This study is preliminary in nature and might have limitations regarding the sample size, methodology and sampling techniques. Therefore, further extensive studies involving more samples and more personnel and utilizing validated methodology and precisely calibrated instruments are essential to get more acceptable and conclusive data.

\section{Acknowledgements}

The authors would like to extend their gratitude towards the University Grants Commission of Bangladesh for providing financial support to complete the research work. The authors are also grateful to Ibn Sina Pharmaceuticals Ltd. for donating LSP and OLM reference standards.

\section{References}

Aniñon, A.D.A., Binos, R.S.R., Brizuela, K.M.M., de Luna, W.J.C., del Rosario, R.P.S., Dimalala, J.J.C., Dueñas, T.J.P., Linatoc, I.I.B., Recto, J.P.D., Salinas, M.V. and Paraiso, W.K.D. 2014. Spectrophotometric determination of losartan potassium in tablets. Sci. Diliman. 26, 41-52.
Carr-Lopez, S.M., Mallett, M.S. and Morse, T. 1995. The tablet splitter: barrier to compliance or cost-saving instrument? Am. J. Health-Syst. Pharm. 52, 27072708.

Celebier, M. and Altinoz, S. 2007. Determination of olmesartan medoxomil in tablets by UV-Vis spectrophotometry. Pharmazie. 62, 419-422.

Ciavarella, A. B., Khan, M. A., Gupta, A., and Faustino, P. J. 2016. Dose uniformity of scored and unscored tablets: Application of the FDA Tablet Scoring Guidance for Industry. PDA J Pharm Sci Tech.70, 523-532.

Gupta, P. and Gupta, K. 1988. Broken tablets: does the sum of the parts equal the whole? Am. J. Hosp. Pharm. 45, 1498-1498.

Kasonde, L., Tordrup, D., Naheed, A., Zeng, W. and Ahmed, S. 2019. Evaluating medicine prices, availability and affordability in Bangladesh using World Health Organization and Health Action International methodology. BMC Health Serv. Res. 19, 383.

Madathilethu, J., Roberts, M., Peak, M., Blair, J., Prescott, R. and Ford, J.L. 2018. Content uniformity of quartered hydrocortisone tablets in comparison with mini-tablets for pediatric dosing. BMJ Paediatr. Open 2, 1-7.

Muir, V.J. and Keating, G.M. 2010. Olmesartan medoxomil. Drugs 70, 2439-2447.

Myriam, S., Philippe, A., Fontan, J.E. and Brion, F. 1994. Splitting tablets in half. Am. J. Health-Syst. Pharm. 15, 548-550.

Nimmi, I., Romana, B., Islam, M.K., Chowdhury, J.A. and Dulal, M.M. 2010. Sectility test on three marketed drugs act on ß-receptor. Bangladesh Pharm. J. 13, 7173.

Ono, A., Nagaoka, N., Marunaka, S. and Nomura, Y. 2013. U.S. Patent 8, 541, 024.

Quinzler, R., Gasse, C., Schneider, A., Kaufmann-Kolle, P., Szecsenyi, J. and Haefeli, W.E. 2006. The frequency of inappropriate tablet splitting in primary care. Eur. J. Clin. Pharmacol. 62, 1065-1073.

Sedrati, M., Arnaud, P., Fontan, J.E. and Brion, F. 1994. Splitting tablets in half. Am. J. Health-Syst. Pharm. 51, 548-549.

Shahinfar, S., Cano, F., Soffer, B.A., Ahmed, T., Santoro, E.P., Zhang, Z., Gleim, G., Miller, K., Vogt, B., Blumer, J. and Briazgounov, I. 2005. A double-blind, dose-response study of losartan in hypertensive children. Am. J. Hypertens. 18, 183-190. 
Stimpel, M., Kuffer, B., Groth, H. and Vetter, W. 1984. Breaking tablets in half. The Lancet, 323, 1299.

Stimpel, M., Vetter, H., Küffer, B., Groth, H., Greminger, P. and Vetter, W. 1985. The scored tablet-a source of error in drug dosing? J. Hypertens. 3, S97-S99.

Teixeira, M.T., Sá-Barreto, L.C., Gratieri, T., Gelfuso, G.M., Silva, I.C. and Cunha-Filho, M.S. 2017. Key technical aspects influencing the accuracy of tablet subdivision. AAPS PharmSciTech. 18, 1393-1401.

Trivedi, M.R., Patel, H.H. and Dave, R.H. 2017. A review on tablet scoring: background, history and current regulatory considerations. J. Pharm. Res. Int. 20, 1-7.
Van Santen, E., Barends, D.M. and Frijlink, H.W. 2002. Breaking of scored tablets: a review. Eur. J. Pharm. Biopharm. 53, 139-145.

Wilson, M.M., Kaiser, F.E. and Morley, J.E. 1996. Tablet breaking ability of older diabetic persons. J. Am. Geriatr. Soc. 44, 106.

Zaid, A.N. and Ghosh, A.A. 2011. Compliance of scored tablet halves produced by Palestinian Pharmaceutical Companies with the new European Pharmacopoeia requirements. Arch. Pharm. Res. 34, 1183. 